\title{
Part 11: User Participation
}

\author{
Co-creation of Assistive Solutions \\ Jorge Gómez Sanz, Rubén Fuentes and Juan Pavón* \\ Facultad de Informática, Universidad Complutense de \\ Madrid, 28040 Madrid, Spain
}

Background: The working hypothesis is that collaboration of experts to define Ambient Assisted Living (AAL) solutions for people with disabilities is often difficult because the lack of a common language that would allow each one to state what is the problem and the solutions from their point of view. The interlingua that is proposed here is the use of videos, which are based on the use of simulations of daily living situations and technological solutions that can help to improve such situations. The communication among participants uses these simulations to support their understanding and discussion of the setting, and participants provide their observations in terms of modifications and annotations on these simulations. This workflow of interactions would facilitate arriving to a consensus on the problem's definition and the assessment of the solutions.

Method: The approach is multi-disciplinary as it involves the participation of different specialists (engineers for implementing the solutions, sociologists, psychologists, social workers) as well as the care givers (in many cases also relatives) and the end users. There is a combination of different methodologies from social sciences and software engineering. The flexibility of the process is supported by model-driven engineering techniques. This allows generating the simulations from graphical models that specify their different aspects (e.g. the activities or the space).

Key results: (1) A web-based collaborative platform that facilitates the discussion and agreement among experts with different profiles. (2) Case studies that will illustrate the use of the platform for modelling disabilities that are related with neurodegenerative processes. (3) Modelling languages that are adapted to experts of different specialties, in order to formalize existing knowledge in AAL that is normally partially recorded.
(4) A catalogue of typical behaviours of persons and simulation elements that can be reused. (5) Guidelines, collaboration recommendations, and examples for using the platform and visual methods for the creation and validation of new scenarios.

Conclusion: The software tools have been distributed as open source and a website shows several case studies: http://grasia.fdi.ucm.es/hackwithpeople/. This platform supports a collaborative development of AAL solutions, with collaboration of all the interested stakeholders (e.g. final users, relatives, caregivers and experts). The use of videos as a mechanism to understand and discuss on the problem and present solutions has shown to be more effective than other traditional engineering tools (e.g., diagrams, technical specifications). Keywords: Ambient Assisted Living, Simulation, Virtual Living Lab, Co-creation.

*Corresponding author. E-mail: jpavon@fdi.ucm.es

\section{Hackcessible: Towards a new model for stimulating user-led innovation in Assistive Technology \\ Aejaz Zahid ${ }^{\mathrm{a}, *}$, Peter Mylon ${ }^{\mathrm{b}}$ and Simon Wheatcroft ${ }^{\mathrm{c}}$ \\ ${ }^{a}$ Innovation Hub, SYB Integrated Care System, 722 Prince of Wales Road, Sheffield, UK \\ ${ }^{\mathrm{b}}$ iForge Makerspace, Faculty of Engineering, The Uni- versity of Sheffield, Sheffield, UK \\ ${ }^{\mathrm{c}}$ Department of Computer Science, The University of Sheffield, Sheffield, UK}

Background: In recent years Co-Design or Participatory design methods have been mentioned by numerous researchers and developers as a preferred framework for product or service design and increasingly by those involved in assistive technology development. With increasing democratization of design and fabrication tools, we are also seeing many instances of assistive technology users themselves innovating solutions for their own needs globally and in many cases sharing these solutions online in an open source manner. Furthermore, hackathons and make-a-thons addressing 
disability needs are gaining popularity the world over as a means to stimulate new innovation in assistive technology by leveraging the skills of the maker community, students and technology developers in a very short and intense timeframe. The authors have participated in and studied numerous models of such concentrated events focused on disability innovation and found significant gaps in need finding, user involvement and post-event sustainability of projects. The authors propose a new model for a disability focused hack/make-a-thon that brings together elements of participatory design and user innovation, along with a framework for sustainable technology \& enterprise development beyond what is typically a weekend long event.

Method used: We present a case study for a hackathon /make-a-thon, along with a carefully curated programme of pre- and post-hackathon workshops that have been designed to both facilitate user led innovation and create opportunities for sustained technology development. In addition, at every stage of the process, we have surveyed participants to assess the effectiveness of this model.

Key results: Under this model over $75 \%$ of participating teams have developed prototype solutions that have been taken forward for either commercialization or as open source assistive technology projects.

Conclusions: By facilitating a collaborative user-led innovation approach between a diverse team of technology developers and technology users, and through a sustained process of co-learning, co-design and cocreation, we have developed a new model for stimulating and accelerating assistive technology innovation in hackathons that results in a greater probability of successful outcomes.

Keywords: Hackathon, Assistive, Co-Design, CoCreation, Participatory

*Corresponding author. E-mail: a.zahid@nhs.net

\author{
Study on How Health Care Service Providers To- \\ gether with Industry Partners Can Co-design Ac- \\ cessible Assistive Technology for Individuals with \\ Intellectual Disabilities (ID) \\ John Owuor ${ }^{\mathrm{a}}$, Sarah Gavra Boland ${ }^{\mathrm{b}, *}$, Aine Walsh ${ }^{\mathrm{c}}$ \\ and Peter Gallagher ${ }^{\mathrm{d}}$ \\ a ASSISTID Fellow, ALL Institute, Maynooth Univer- \\ sity, County Kildare, Ireland \\ ${ }^{\mathrm{b}}$ Liffey Services, John of God Community Services clg. \\ Cookstown Way, Tallaght, Dublin 24, Ireland \\ ${ }^{\mathrm{d}}$ Saint John of God Hospitaller Services, Stillorgan, \\ Co. Dublin, Ireland.
}

Background: The global drive to promote inclusion of people with intellectual disability (ID) into community living has had significant progress in the educational, social and occupational fields. The availability of quality digital education has been suggested in the literature to enhance the access of adults with ID to societal goods and their rights, enabling them to become active citizens. However only 1 in 10 people with disability who need assistive technology (AT) has access globally. For people with intellectual disability (ID), the proportion of those who need AT that have access remains unknown. This study aimed to offer evidence on how AT can be co-designed between health care service providers and technology industry partners to support social inclusion for people with ID. The study looks at an Erasmus+ funded Project DESKTOP with six EU country service providers working with Fundación Vodafone España, to co-design and build an accessible app Mefacilyta, to assist independence with diverse and transitioning service providers.

Method: The study used a cross-sectional comparative qualitative investigation focusing on the central issue of the role of AT in facilitating social inclusion and boosting equality for people with ID. Data were collected using face to face interviews between January to July 2017. Observations were used to complement any verbal data gathered through interviews. The study involved participants with diverse communication abilities, making it impossible to solely rely on interviews hence the use of mixed methods. Literature review was used to synthesize existing knowledge on how AT enhances social inclusion (access to services and interpersonal relationships)

Key results: The Mefacilyta App enabled the users to improve their ability to carry out various tasks with reduced support requirements. AT was seen to significantly empower its users to improve their independence and wellbeing. It was important to see how open the technology builder, Fundación Vodafone España, were to engage with the end users of the tool to ensure it was suitable for all. Participants in the present study were not only able to use the App to learn life skills but were also involved in how to improve the app to enhance usability enabling them to perform tasks that would have previously relied on support from others. One example how the users influenced the tool can be seen in the adoption of a more accessible calendar feature that now has text to speech integration. These additional features supported the autonomy of the users, it was a great boost for their self-esteem, sense of wellbeing and community inclusion. 
Conclusion: Assistive technology can support inclusion for people with ID, particularly if there is collaborative co-development between all relevant stakeholders to ensure needs-based solutions. But access and usage barriers need to be overcome. Technology developers need to work directly with people with disabilities to co-design accessible solutions. Further research is needed to explore how best to ensure stakeholder engagement particularly how-to co-design with people with severe levels of ID.

Keywords: Co-Design, Assistive Technology, digital divide, user centered design.

*Corresponding author. E-mail: sarah.boland@sjog.ie

\section{Assistive Technology Users at the Coal Face of Con- fronting Intrinsic Design Issues in their Assistive Technology \\ Bernard Timmins ${ }^{\mathrm{a}, *}$, Siobhan Long $^{\mathrm{b}}$ and Lorraine D'Arcy ${ }^{\mathrm{c}}$ \\ ${ }^{\text {a }}$ Lecturer at School of Mechanical \& Design Engineer- ing, City Campus TU Dublin, Dublin, Ireland \\ ${ }^{\mathrm{b}}$ National Manager, Assistive Technology and Seat- Tech services Enable Ireland \\ ${ }^{\mathrm{c}}$ Lecturer at School of Civil and Structural Engineer- ing, City Campus TU Dublin, Dublin, Ireland}

Background: At the Technological University of Dublin a Product Design programme students, and members of the Disabled Community (Assistive Technology users) have teamed together to create a unique multidisciplinary learning environment for Assistive Technology and Universal Design. Finally, this paper will show the outcomes of various student feedbacks highlighting the observed benefits of this approach.

Method: Having discussed design with AT Users students realise very quickly that AT devices are not the 'panacea for all ills'. Indeed the challenges of using 'off-the-shelf' generic AT devices becomes immediately clear, as people with varying degrees of disability experience difficulty adapting to and using these generic devices. Remarkably there is a reported abandonment rate of $78 \%$ for certain AT technologies. Students are encouraged to keep this notion of abandonment very much in mind when they are developing their design concept, and they are encouraged to (and supported by Enable Ireland) engage with both clients and staff, to seek their feedback, on their emerging designs. The students have complete freedom with the AT design, but are expected to draw upon theoretical concepts delivered in class in order to support their ideas. It is expected that the design will be responsive to client's needs.

Key results: An important aspect of this multidisciplinary approach is that everyone involved recognises that people with disabilities have the same aspirations to independence as their peers, this partnership sought to address the inequalities that exist in some product design which limit their use, and to identify novel design concepts which would enhance autonomy and independence for participants and for the wider community. interact extremely well with clients, and develop good working relationships. Clients report favourably on students, and enjoy the special attention that the project confers upon them. As a result, successive groups of students are encouraged by their community engagement and have been inspired towards community engagement/design after graduation. During a survey the students answered the following question: "How much has this experience changed your perceptions of design?" The mode response was $100 \%$ and the average response was $83 \%$. In this journey from theory to practice, through engagement with clients, students learn about responsive design.

Conclusion: This approach has outcome and impact, both on the student and the community that is pervasive and far-reaching. While on the one hand the outcomes of this element of the module appear modest (product design), the enactment of this results in a powerful and emotive journey for the student, one that not only promotes learning in the real world, and engages and empowers community partners, but has fostered the development of influential partnership across industry and practice. Through the fostering of community relations, and building relationships between industry and the third level organisation that this module has created, pathways have been developed that pave the way for the Engineering community to positively engage with AT design and national disability services that can have far-reaching consequences and raise awareness of the need for Universal Design.

Keywords: Universal Design, Community, Engagement, Assistive Technology, Enable Ireland.

*Corresponding author. E-mail: bernard.timmins@ dit. ie

\footnotetext{
Enabling User-centered Design and Evaluation to Increase Acceptance of Wearable Robotic Assistive Technologies

Jan T. Meyer ${ }^{\mathrm{a}, *}$, Olivier Lambercy ${ }^{\mathrm{a}}$ and Roger Gassert $^{\mathrm{a}}$
} 
${ }^{a}$ Department of Health Sciences and Technology, ETH Zurich, Switzerland

Background: With wearable, lightweight and more affordable robotics on the rise, new opportunities for daily life assistance for people with neuromuscular disorders become available. However, the successful application of a device stands and falls with the respective user experience and technology acceptance. Unfortunately, the majority of promising research prototypes underestimate these obstacles or fail to consider the human factors confounding assistive technology usage. User-centered design (UCD) of assistive technologies has shown to remarkably increase user experience. An intensified target population interaction with early prototypes can potentially provide valuable insights to overcome the gap between in-lab testing and clinical or home application. However, understanding and analyzing user experience is not yet sufficiently established, lacking clear guidelines and standardized measures. This generates difficulties to define requirements and assess device performance, especially in the emerging area of wearable robotics. There is a need to simplify and enable the user-centered evaluation and design of assistive technologies to successfully tackle the prominent acceptance limitations.

Method: We propose a UCD framework for wearable robotic assistive technologies, addressing three specific aspects currently limiting the success of UCD design processes: (i) planning and preparation, (ii) context and needs assessment and (iii) user testing and evaluation. To understand the current limitations of UCD, surveys are being conducted with development experts, health care professionals, caregivers as well as end-users and their next of kin. With this collection and repository of knowledge, an improved understanding and assessment of user needs in the context of device usage can be proposed. Through contextualization, we define required stakeholder involvement milestones during development and achieve a more targeted selection of user experience measures. A UCD toolbox selecting and proposing standardized outcome measures from an online database is currently being established, with the aim to define comparable evaluation protocols with appropriate measures fitting the context of use. A preliminary version of the UCD framework was tested to evaluate and redesign the physical human-robot interface of the VariLeg powered lower limb exoskeleton, involving two experienced paraplegic users.

Key results: The UCD framework under development aims to provide standardized, context-specific outcome measures to (a) assess end-user experiences (b) identify key limitations and (c) provide more generalizable usability results when testing wearable robotic assistive technologies. The enhanced feedback from the evaluation phase can be used to further refine the context and needs assessment and initiate the next design cycle. In our research with the VariLeg gait exoskeleton, this has shown to facilitate target population involvement and motivated the iterative design of individual interface components, leading to an increase of $45 \%$ in the perceived usability, measured by the System Usability Scale (SUS).

Conclusion: Acceptance limitations of novel assistive technologies are aimed to be tackled with a simplified UCD approach. Maximizing user experience relative to the context of use can help to close the gap between in-lab testing of research prototypes and real-life application in daily usage. This can not only increase development efficiency but, more importantly, increase the quality of life of those in need of technological support. Keywords: User-centered Design, Assistive Technology, Wearable Robotics, Usability Evaluation *Corresponding author. E-mail: jan.meyer@ hest.ethz. ch

\section{Benefits Beyond Experience}

Vivienne Conway* and Amanda Mace Web Key IT Pty Ltd, 10 Tonrita Place, Wanneroo, WA 6065, Australia

Background: Many organisations provide digital accessibility services. Management generally view accessibility as benefiting a handful of their user group. They question the 'Return on Investment' on money spent on accessibility compliance. When an organisation truly embraces accessibility, not only do we meet local legislative and International human rights obligations, but we reduce costs, and build our internal capacity for design and innovation. Clients become our partners in building for universal design and build partnerships with their customers, continually creating delightful user experiences. In our observation of various organisations and their approach, we have located a set of characteristics of the organisation taking the road less travelled to embrace accessibility organisationwide.

Method used: Our work is observational, based upon experience with a broad range of clients from federal government to not-for-profit. Using our Digital Accessibility Maturity Model (DAMM), and working with organisations we assess their placement on the DAMM 
from 0 - no knowledge/application to 5 - embed digital accessibility into all projects, products, processes and business management. The characteristics and experience of the mature organisation demonstrate there are benefits in becoming accessible beyond that of the practical interaction of the user with disabilities.

Key results: The organisation placing highly on the DAMM looks beyond a technical compliance/checklist mentality. Compliance is only as good as your ability to ensure that the philosophy is embraced and all new content is tested to ensure compliance. There will always be issues out of your control - such as usergenerated content and difficulty locating accessible third-party products. The mature organisation incorporates best practices into its culture and practice, making it part of their standard operating procedure. They prioritize people with disabilities when making design decisions. Accessibility is integrated into training and education programs. They test to ensure that users of assistive technology are able to have a seamless experience, as equivalent as possible to that of the person without disability. When accessibility is viewed by the mature organisation, they see benefits beyond that of the user's individual experience. When a user has a great (accessible) experience, it is not just the fact that they are able to accomplish a task. One example is the user tester who had such a good experience with a local government website she stated "it made me want to move there". There are benefits beyond user experience, such as public relations, customer referral, leadership and reputation. The organisation approaching the more mature end of the continuum by incorporating diversity into their hiring practices, ensures all digital material is accessible, and looks for ways to show leadership.

Conclusion: The more mature organisation, sees the benefit of incorporating the needs of all of their users into their design as well as into other aspects of their organisation. They realize that meeting the needs of people with disabilities allows them to create a better experience for all of users. They approach accessibility as an opportunity to create a new and more satisfying type of interaction with their user - one that goes beyond compliance with a standard.

*Corresponding author. E-mail: v.conway@webkeyit. com 